International Journal of Pure and Applied Mathematics

Volume 106 No. $1 \quad 2016,127-139$

ISSN: 1311-8080 (printed version); ISSN: 1314-3395 (on-line version)

url: http://www.ijpam.eu

doi: 10.12732/ijpam.v106i1.10

ijpam.eu

\title{
ON THE GERBER-SHIU PENALTY FUNCTION FOR THE COMPOUND BINOMIAL RISK MODEL WITH DELAYED CLAIMS
}

\author{
Zhenhua $\mathrm{Bao}^{1}{ }^{\S}, \mathrm{He} \mathrm{Liu}^{2}, \mathrm{Ye} \mathrm{Liu}^{3}$ \\ ${ }^{1,3}$ School of Mathematics \\ Liaoning Normal University \\ Dalian, 116029, P.R. CHINA \\ ${ }^{2}$ School of Mathematics, \\ Physics and Biological Engineering \\ Inner Mongolia University of Science and Technology \\ Baotou, 014010, P.R. CHINA
}

\begin{abstract}
In this paper we consider the Gerber-Shiu penalty function in the compound binomial risk model with time-correlated claims. It is assumed that each main claim will induce a by-claim but the occurrence of the by-claim may be delayed with a certain probability. Formulas for the probability generating function of the penalty function are obtained, together with the expression for the penalty function with zero initial surplus. Then we show that the penalty function satisfies a defective renewal equation. When the claims follow geometric distributions, explicit expression for the Gerber-Shiu function is derived. Numerical example is provided to illustrate the application of the result discussed in the paper.
\end{abstract}

AMS Subject Classification: 62P05, 91B30

Key Words: main claim, by-claim, probability generating function, Gerber-shiu function, defective renewal equation

\section{Introduction}

The classical compound binomial risk model has been studied in actuarial lit-

Received: August 28, 2015

Published: February 2, 2016

$\S_{\text {Correspondence author }}$
(C) 2016 Academic Publications, Ltd.

url: www.acadpubl.eu 
erature by $[1,2,3]$ among many others. Some ruin quantities such as ruin probabilities, the deficit at ruin and the surplus prior to ruin are investigated extensively, based on the assumption of stationary and independent increment in the surplus process. In practical contexts, this assumption can be restrictive. Recently, many authors consider the compound binomial risk model with time-correlated claims. Yuen and Guo [4] present a compound binomial model by assuming that every main claim will produce a by-claim but the occurrence of the by-claim may be delayed for one time period with a certain probability. They derive recursive formulas for the finite time ruin probabilities and explicit expressions for ultimate ruin probabilities. Xiao and Guo [5] further investigate the joint distribution of the surplus immediately prior to ruin and deficit at ruin. $\mathrm{Wu}$ and $\mathrm{Li}[6]$ consider a compound binomial model with time-correlated individual claims and dividend payments that are ruled by a constant dividend barrier, the expected present value of total dividends are studied. Other dependence structures in terms of main claims and by-claims are studied in $\mathrm{Wu}$ and Yuen [7] and the references therein.

Motivated by Yuen and Guo [4], we explore the compound binomial model with different framework of time-correlated claims. It is assumed that each main claim induces a by-claim. The number of main claim process $\{N(n), n \in \mathbb{N}\}$ is a binomial process with independent and identically distributed (i.i.d.) interclaim times $\left\{T_{1}, T_{2}, \ldots\right\}$ having probability function (p.f.) $k(x)=(1-q) q^{x-1}$ for $x \in$ $\mathbb{N}^{+}$and $0<q<1$. The main claim amounts $\left\{X_{1}, X_{2}, \ldots\right\}$ are i.i.d. positive and integer valued random variables with common cumulative distribution function (c.d.f.) $F(x)=1-\bar{F}(x)$, p.f. $f(x)$ and mean $\mu_{F}$.

Suppose that the main claim $X_{i}$ in epoch $T_{i}$ will induce a by-claim $Y_{i}$. Moreover, the by-claim $Y_{i}$ may occur simultaneously with main claim $X_{i}$ with probability $\theta(0 \leq \theta \leq 1)$, or the occurrence of the by-claim may be delayed to $T_{i+1}$ with probability $1-\theta$. In the later case, we assume that the occurrence of the delayed by-claim $Y_{i}$ is independent of the occurrence of the next main claim $X_{i+1}$.

The by-claims $\left\{Y_{1}, Y_{2}, \ldots\right\}$ are also i.i.d. positive and integer valued random variables having c.d.f., p.f. and mean denoted by $G(y), g(y)$ and $\mu_{G}$, respectively. The independence between $\left\{X_{1}, X_{2}, \ldots\right\},\left\{Y_{1}, Y_{2}, \ldots\right\}$ and $\{N(n), n \in$ $\mathbb{N}\}$ are assumed. Suppose that the premium rate received per period is 1 , then the surplus process $U(n)$ of an insurance company at time $n$ is described as

$$
U(n)=u+n-\sum_{i=1}^{N(n)} X_{i}-R(n), \quad n \in \mathbb{N},
$$

where $U(0)=u \in \mathbb{N}$ is the initial surplus and $R(n)$ is the total by-claim amounts 
up to time $n$. With $p=1-q$, it is easy to see that

$$
\mathrm{E}\left(\sum_{i=1}^{N(n)} X_{i}+R(n)\right)^{n}=n p \mu_{F}+n p \mu_{G}-(1-\theta) \mu_{G}\left(1-q^{n}\right) .
$$

Therefore, we further assume that $p\left(\mu_{F}+\mu_{G}\right)<1$, providing a positive loading condition.

Let $T=\inf \left\{n \in \mathbb{N}^{+}: U(n)<0\right\}$ be the time of ruin with $T=\infty$ if ruin does not occur. Note that if ruin occurs, $U(T-1)$ is the surplus one period prior to ruin and $|U(T)|$ is the deficit at ruin. Denote by

$$
m(u)=\mathrm{E}[\omega(U(T-1),|U(T)|) I(T<\infty) \mid U(0)=u]
$$

the Gerber-Shiu expected discounted free penalty function (e.g., Gerber and Shiu [8]). Where $\omega\left(u_{1}, u_{2}\right): \mathbb{N} \times \mathbb{N}^{+} \rightarrow \mathbb{N}$ and $I(A)$ is the indicator function of an event $A$.

The rest of the paper is structured as follows. In Section 2, both the GerberShiu penalty function with zero initial surplus and the generating function of the penalty function are obtained. The defective renewal equation satisfied by the penalty function is derived in Section 3. In Section 4 the explicit expression for the penalty function is given when the individual claim amounts from both classes follow geometric distribution. Numerical result is also provided to illustrate the application of the result discussed in this section.

\section{The Probability Generating Function}

Throughout the rest of the entire paper, unless otherwise stated, we adopt the convention that $\sum_{m}^{n}=0$ when $n<m$. For any function $a(x)$, we will use $\hat{a}(z)$ to denote the corresponding probability generating function (p.g.f.).

In order to derive the p.g.f. of the expected penalty function $m(u)$, we consider a complementary surplus process as follows. Instead of having one main claim and a by-claim with probability $\theta$ in the first epoch $T_{1}$, another by-claim distributed as $Y_{1}$ occurs simultaneously at this time. We denote the corresponding expected discounted free Gerber-Shiu function for this auxiliary model by $m_{1}(u)$. For the risk process $(1)$, there are two possibilities at time $T_{1}$ :

1. A main claim and its by-claim occur concurrently, then the surplus process gets renewed except for the initial value; 
2. A main claim occurs and its associated by-claim is delayed to $T_{2}$, then the surplus process transfers to the auxiliary model described as above.

Considering what will happen at time $T_{1}$, by the law of total probability we have

$$
m(u)=p \sum_{t=1}^{\infty} q^{t-1}\left[\theta \gamma(u+t)+(1-\theta) \gamma_{1}(u+t)\right]
$$

where

$$
\begin{aligned}
& \gamma(i)=\sum_{k=2}^{i} m(i-k) f * g(k)+\omega(i), \quad \omega(i)=\sum_{k=i+1}^{\infty} \omega(i-1, k-i) f * g(k), \\
& \gamma_{1}(i)=\sum_{k=1}^{i} m_{1}(i-k) f(k)+\omega_{1}(i), \quad \omega_{1}(i)=\sum_{k=i+1}^{\infty} \omega(i-1, k-i) f(k),
\end{aligned}
$$

for $i \in \mathbb{N}^{+}$, and $*$ denotes convolution of the p.f.s. Equation (2) can be rewritten as

$$
q m(u)=p \sum_{t=1}^{\infty} q^{t}\left[\theta \gamma(u+t)+(1-\theta) \gamma_{1}(u+t)\right]
$$

But from (2) with $u$ replaced by $u-1$, it follows that

$$
m(u-1)=p \sum_{t=0}^{\infty} q^{t}\left[\theta \gamma(u+t)+(1-\theta) \gamma_{1}(u+t)\right], \quad u \in \mathbb{N}^{+}
$$

Subtracting (3) from (4) yields

$$
m(u-1)-q m(u)=p\left[\theta \gamma(u)+(1-\theta) \gamma_{1}(u)\right], \quad u \in \mathbb{N}^{+} .
$$

Multiplying (5) by $z^{u}$ and summing over $u$ from 1 to $\infty$, it follows that

$$
(z-q) \hat{m}(z)+q m(0)=p\left\{\theta \hat{m}(z) \hat{f}(z) \hat{g}(z)+(1-\theta) \hat{m}_{1}(z) \hat{f}(z)+\hat{\eta}(z)\right\}
$$

which is equivalent to

$$
[z-q-p \theta \hat{f}(z) \hat{g}(z)] \hat{m}(z)-p(1-\theta) \hat{f}(z) \hat{m}_{1}(z)=p \hat{\eta}(z)-q m(0),
$$

where $\hat{\eta}(z)=\theta \hat{\omega}(z)+(1-\theta) \hat{\omega}_{1}(z)$.

For the auxiliary process we have analogously

$$
m_{1}(u)=p \sum_{t=1}^{\infty} q^{t-1}\left\{\theta \gamma_{2}(u+t)+(1-\theta) \gamma_{3}(u+t)\right\},
$$


where

$$
\begin{aligned}
\gamma_{2}(i) & =\sum_{k=3}^{i} m(i-k) f * g * g(k)+\omega_{2}(i), \\
\omega_{2}(i) & =\sum_{k=i+1}^{\infty} \omega(i-1, k-i) f * g * g(k), \\
\gamma_{3}(i) & =\sum_{k=2}^{i} m_{1}(i-k) f * g(k)+\omega(i),
\end{aligned}
$$

for $i \in \mathbb{N}^{+}$. Imitating closely the procedure to derive the Equation (7), we have

$$
[z-q-p(1-\theta) \hat{f}(z) \hat{g}(z)] \hat{m}_{1}(z)-p \theta \hat{f}(z) \hat{g}^{2}(z) \hat{m}(z)=p \hat{\eta}_{1}(z)-q m_{1}(0),
$$

where $\hat{\eta}_{1}(z)=\theta \hat{\omega}_{2}(z)+(1-\theta) \hat{\omega}(z)$.

Solving the simultaneous Equations (7) and (8), we obtain the expressions for $\hat{m}(z)$ and $\hat{m}_{1}(z)$ as follows:

$$
\hat{m}(z)=\frac{\left\{\begin{array}{c}
(z-q-p(1-\theta) \hat{f}(z) \hat{g}(z))(p \hat{\eta}(z)-q m(0)) \\
+p(1-\theta) \hat{f}(z)\left(p \hat{\eta}_{1}(z)-q m_{1}(0)\right)
\end{array}\right\}}{(z-q)[z-q-p \hat{f}(z) \hat{g}(z)]}
$$

and

$$
\hat{m}_{1}(z)=\frac{\left\{\begin{array}{c}
(z-q-p \theta \hat{f}(z) \hat{g}(z))\left(p \hat{\eta}_{1}(z)-q m_{1}(0)\right) \\
+p \theta \hat{f}(z) \hat{g}^{2}(z)(p \hat{\eta}(z)-q m(0))
\end{array}\right\}}{(z-q)[z-q-p \hat{f}(z) \hat{g}(z)]} .
$$

To determine the constants $m(0)$ and $m_{1}(0)$, we consider the roots of the common denominator of (9) and (10), i.e., the roots of the following equation:

$$
(z-q)[z-q-p \hat{f}(z) \hat{g}(z)]=0 .
$$

Obviously, 1 and $q$ are two roots to the Equation (11). Now let $l(z)=z-q$ and $\kappa(z)=p \hat{f}(z) \hat{g}(z)$. It is easy to see that $\kappa^{\prime}(z) \geq 0, \kappa^{\prime \prime}(z) \geq 0$. Also, $l(q)=0<$ $\kappa(q)$ and $l(1)=1-q=p=\kappa(1)$. Furthermore, $\left.\kappa^{\prime}(z)\right|_{z=1}=p\left(\mu_{F}+\mu_{G}\right)<1$ by noting the safety loading condition. Therefore, we conclude that 1 and $q$ are unique roots to the equation (11) in the interval $(0,1]$.

Since $\hat{m}(z)$ and $\hat{m}_{1}(z)$ are analytic, 1 and $q$ must be zeros of the numerators of (9) and (10). Then we can obtain the expressions for $m(0)$ and $m_{1}(0)$ after some calculations

$$
m(0)=\frac{p\left\{\theta \hat{\eta}(1)+(1-\theta)\left[\hat{g}(q) \hat{\eta}(q)+\hat{\eta}_{1}(1)-\hat{\eta}_{1}(q)\right]\right\}}{q[\theta+(1-\theta) \hat{g}(q)]}
$$


and

$$
m_{1}(0)=\frac{p\left\{(1-\theta) \hat{\eta}_{1}(1) \hat{g}(q)+\theta\left[\hat{g}(q)(\hat{\eta}(1)-\hat{\eta}(q))+\hat{\eta}_{1}(q)\right]\right\}}{q[\theta+(1-\theta) \hat{g}(q)]} .
$$

Hence, $\hat{m}(z)$ and $\hat{m}_{1}(z)$ can be determined by substituting (12) and (13) into (9) and (10) respectively.

In the sequel, we denote the two distinct roots to (11) by $\rho_{1}=1$ and $\rho_{2}=q$ for notational convenience.

\section{The Defective Renewal Equation}

In this section we aim to derive the defective renewal equation for the GerberShiu function $m(u)$. In order to simplify calculations, we will use the discrete operator proposed by $\mathrm{Li}[9]$. More precisely, define $T_{r}$ to be the operator of any real valued function $\xi(x), x \in \mathbb{N}^{+}$, by

$$
T_{r} \xi(y)=\sum_{x=y}^{\infty} r^{x-y} \xi(x), \quad y \in \mathbb{N}^{+}
$$

Some nice properties for this operator have been given in [9].

Now we denote the denominator of $(9)$ by $\hat{h}_{1}(z)-\hat{h}_{2}(z)$, where $\hat{h}_{1}(z)=$ $(z-q)^{2}$ and $\hat{h}_{2}(z)=p(z-q) \hat{f}(z) \hat{g}(z)$. Let $h_{1}(x)$ and $h_{2}(x)$ are the inverse function of $\hat{h}_{1}(z)$ and $\hat{h}_{2}(z)$, respectively. Using the Lagrange interpolating polynomial, $\hat{h}_{1}(z)$ can be rewritten as

$$
\begin{aligned}
\hat{h}_{1}(z)= & \hat{h}_{1}(0) \frac{\left(z-\rho_{1}\right)\left(z-\rho_{2}\right)}{\rho_{1} \rho_{2}}+\hat{h}_{1}\left(\rho_{1}\right) \frac{z\left(z-\rho_{2}\right)}{\rho_{1}\left(\rho_{1}-\rho_{2}\right)}+\hat{h}_{1}\left(\rho_{2}\right) \frac{z\left(z-\rho_{1}\right)}{\rho_{2}\left(\rho_{2}-\rho_{1}\right)} \\
= & \hat{h}_{1}(0) \frac{\left(z-\rho_{1}\right)\left(z-\rho_{2}\right)}{\rho_{1} \rho_{2}}+\hat{h}_{2}\left(\rho_{1}\right) \frac{z-\rho_{2}}{\rho_{1}-\rho_{2}}+\hat{h}_{2}\left(\rho_{2}\right) \frac{z-\rho_{1}}{\rho_{2}-\rho_{1}} \\
& +\left(z-\rho_{1}\right)\left(z-\rho_{2}\right)\left(\frac{\hat{h}_{2}\left(\rho_{1}\right)}{\rho_{1}\left(\rho_{1}-\rho_{2}\right)}+\frac{\hat{h}_{2}\left(\rho_{2}\right)}{\rho_{2}\left(\rho_{2}-\rho_{1}\right)}\right)
\end{aligned}
$$

Therefore,

$$
\begin{aligned}
\hat{h}_{1}(z)-\hat{h}_{2}(z)= & \left(z-\rho_{1}\right)\left(z-\rho_{2}\right)\left(\frac{\hat{h}_{1}(0)}{\rho_{1} \rho_{2}}+\frac{\hat{h}_{1}\left(\rho_{1}\right)}{\rho_{1}\left(\rho_{1}-\rho_{2}\right)}+\frac{\hat{h}_{1}\left(\rho_{2}\right)}{\rho_{2}\left(\rho_{2}-\rho_{1}\right)}\right) \\
& -\left(\hat{h}_{2}(z)+\frac{\left(z-\rho_{1}\right) \hat{h}_{2}\left(\rho_{2}\right)-\left(z-\rho_{2}\right) \hat{h}_{2}\left(\rho_{1}\right)}{\rho_{1}-\rho_{2}}\right) .
\end{aligned}
$$


By noting that $\rho_{1}=1$, it is easy to see that

$$
\frac{\hat{h}_{1}(0)}{\rho_{1} \rho_{2}}+\frac{\hat{h}_{1}\left(\rho_{1}\right)}{\rho_{1}\left(\rho_{1}-\rho_{2}\right)}+\frac{\hat{h}_{1}\left(\rho_{2}\right)}{\rho_{2}\left(\rho_{2}-\rho_{1}\right)}=1 .
$$

On the other hand, by the properties of the discrete operator, we obtain

$$
\begin{aligned}
& \hat{h}_{2}(z)+\frac{\left(z-\rho_{1}\right) \hat{h}_{2}\left(\rho_{2}\right)-\left(z-\rho_{2}\right) \hat{h}_{2}\left(\rho_{1}\right)}{\rho_{1}-\rho_{2}} \\
& =\left(z-\rho_{1}\right)\left(z-\rho_{2}\right)\left[T_{z} T_{\rho_{2}} T_{\rho_{1}} h_{2}(1)-T_{z} T_{\rho_{2}} h_{2}(1)\right] .
\end{aligned}
$$

Equations (14)-(17) imply that

$$
\hat{h}_{1}(z)-\hat{h}_{2}(z)=\left(z-\rho_{1}\right)\left(z-\rho_{2}\right)\left[1+T_{z} T_{\rho_{2}} h_{2}(1)-T_{z} T_{\rho_{2}} T_{\rho_{1}} h_{2}(1)\right] .
$$

Now we deal with the numerator of (9). Define

$$
\begin{aligned}
\hat{\tau}_{1}(z)= & q m(0)(z-q), \\
\hat{\tau}_{2}(z)= & p\left\{[z \hat{\eta}(z)-q \hat{\eta}(z)]+p(1-\theta)\left[\hat{Q}_{2}(z)-\hat{Q}_{1}(z)\right]\right. \\
& \left.+q(1-\theta)\left[\hat{f}(z) \hat{g}(z) m(0)-\hat{f}(z) m_{1}(0)\right]\right\},
\end{aligned}
$$

where $\hat{Q}_{1}(z)=\hat{f}(z) \hat{g}(z) \hat{\eta}(z)$ and $\hat{Q}_{2}(z)=\hat{f}(z) \hat{\eta}_{1}(z)$.

Noting that $\hat{\tau}_{2}(z)-\hat{\tau}_{1}(z)$ is an alternative expression for the numerator of (9), $\hat{\tau}_{2}\left(\rho_{i}\right)=\hat{\tau}_{1}\left(\rho_{i}\right)$ holds for $i=1,2$. Since $\hat{\tau}_{1}(z)$ is a polynomial of degree 1 , using the Lagrange interpolating formula once again, we have

$$
\hat{\tau}_{1}(z)=\frac{\hat{\tau}_{2}\left(\rho_{1}\right)\left(z-\rho_{2}\right)-\hat{\tau}_{2}\left(\rho_{2}\right)\left(z-\rho_{1}\right)}{\rho_{1}-\rho_{2}} .
$$

Consequently, by (19) we get

$$
\hat{\tau}_{2}(z)-\hat{\tau}_{1}(z)=\left(z-\rho_{1}\right)\left(z-\rho_{2}\right)\left[T_{z} T_{\rho_{2}} T_{\rho_{1}} \tau_{2}(1)-T_{z} T_{\rho_{2}} \tau_{2}(1)\right]
$$

By (18) and (20) we can rewrite the expression $\hat{m}(z)$ as follows

$$
\hat{m}(z)=\frac{\hat{\tau}_{2}(z)-\hat{\tau}_{1}(z)}{\hat{h}_{1}(z)-\hat{h}_{2}(z)}=\frac{T_{z} T_{\rho_{2}} T_{\rho_{1}} \tau_{2}(1)-T_{z} T_{\rho_{2}} \tau_{2}(1)}{1+T_{z} T_{\rho_{2}} h_{2}(1)-T_{z} T_{\rho_{2}} T_{\rho_{1}} h_{2}(1)},
$$

and (21) implies that

$$
\hat{m}(z)=\left[T_{z} T_{\rho_{2}} T_{\rho_{1}} h_{2}(1)-T_{z} T_{\rho_{2}} h_{2}(1)\right] \hat{m}(z)+\left[T_{z} T_{\rho_{2}} T_{\rho_{1}} \tau_{2}(1)-T_{z} T_{\rho_{2}} \tau_{2}(1)\right] .
$$

Denote by $Q_{1}(k)$ and $Q_{2}(k)$ the inverse image of $\hat{Q}_{1}(z)$ and $\hat{Q}_{2}(z)$ respectively, then we can show that the Geber-Shiu function $m(u)$ satisfies a defective renewal equation. 
Theorem. For $u \in \mathbb{N}$, we have

$$
m(u)=p \sum_{k=0}^{u} m(u-k) \zeta(k)+\varphi(u)
$$

where

$$
\begin{gathered}
\zeta(k)=\sum_{j=k+1}^{\infty} f * g(j) \\
\varphi(u)=p \delta(u)+q(1-\theta) \sum_{k=u+1}^{\infty}\left(1-q^{k-u-1}\right)\left[m(0) f * g(k)-m_{1}(0) f(k)\right], \\
\delta(u)=\sum_{k=u+1}^{\infty}\left\{\eta(k)+(1-\theta)\left(1-q^{k-u-1}\right)\left[Q_{2}(k)-Q_{1}(k)\right]\right\}
\end{gathered}
$$

and $m(0)$ and $m_{1}(0)$ are determined by (12) and (13), respectively.

Proof. For notational convenience, let $\hat{b}(z)=\hat{f}(z) \hat{g}(z)$ and $b(x)$ is the inverse function of $\hat{b}(z)$. Then $\hat{h}_{2}(z)$ can be expressed as $\hat{h}_{2}(z)=p[z \hat{b}(z)-q \hat{b}(z)]$. Thus we have

$$
\begin{aligned}
T_{z} T_{\rho_{2}} T_{\rho_{1}} h_{2}(1)= & \frac{p}{\rho_{1}-\rho_{2}}\left\{\rho_{1} \frac{z \hat{b}(z)-\rho_{1} \hat{b}\left(\rho_{1}\right)}{z-\rho_{1}}-\rho_{2} \frac{z \hat{b}(z)-\rho_{2} \hat{b}\left(\rho_{2}\right)}{z-\rho_{2}}\right. \\
& \left.-q\left(\rho_{1} T_{z} T_{\rho_{1}} b(1)-\rho_{2} T_{z} T_{\rho_{2}} b(1)\right)\right\} .
\end{aligned}
$$

For $i=1,2$, one deduces

$$
\frac{z \hat{b}(z)-\rho_{i} \hat{b}\left(\rho_{i}\right)}{z-\rho_{i}}=\hat{b}(z)+\rho_{i} T_{z} T_{\rho_{i}} b(1)
$$

Substituting (26) into (25) yields

$$
T_{z} T_{\rho_{2}} T_{\rho_{1}} h_{2}(1)=p\left[\hat{b}(z)+\rho_{1} T_{z} T_{\rho_{1}} b(1)\right] .
$$

Similarly, by (26) we have

$$
T_{z} T_{\rho_{2}} h_{2}(1)=p\left\{\frac{z \hat{b}(z)-\rho_{2} \hat{b}\left(\rho_{2}\right)}{z-\rho_{2}}-q \frac{\hat{b}(z)-\hat{b}\left(\rho_{2}\right)}{z-\rho_{2}}\right\}=p \hat{b}(z),
$$


which together with (27) gives

$$
T_{z} T_{\rho_{2}} T_{\rho_{1}} h_{2}(1)-T_{z} T_{\rho_{2}} h_{2}(1)=p \rho_{1} T_{z} T_{\rho_{1}} b(1)
$$

If we denote by $\hat{W}(z)=p(z \hat{\eta}(z)-q \hat{\eta}(z))$ and $W(x)$ the corresponding inverse function, then (28) implies that

$$
T_{z} T_{\rho_{2}} T_{\rho_{1}} W(1)-T_{z} T_{\rho_{2}} W(1)=p \rho_{1} T_{z} T_{\rho_{1}} \eta(1) .
$$

On the other hand, it is easy to see that

$$
\begin{gathered}
T_{z} T_{\rho_{2}} T_{\rho_{1}} Q_{i}(1)-T_{z} T_{\rho_{2}} Q_{i}(1)=\frac{T_{z} T_{\rho_{2}} Q_{i}(1)-T_{z} T_{\rho_{1}} Q_{i}(1)}{\rho_{2}-\rho_{1}}, \quad i=1,2, \\
T_{z} T_{\rho_{2}} T_{\rho_{1}} f(1)-T_{z} T_{\rho_{2}} f(1)=\frac{T_{z} T_{\rho_{2}} f(1)-T_{z} T_{\rho_{1}} f(1)}{\rho_{2}-\rho_{1}} \\
T_{z} T_{\rho_{2}} T_{\rho_{1}} f * g(1)-T_{z} T_{\rho_{2}} f * g(1)=\frac{T_{z} T_{\rho_{2}} f * g(1)-T_{z} T_{\rho_{1}} f * g(1)}{\rho_{2}-\rho_{1}} .
\end{gathered}
$$

Recall the fact that $\rho_{1}-\rho_{2}=p$, combine (30)-(32) leads to

$$
\begin{aligned}
T_{z} T_{\rho_{2}} T_{\rho_{1}} \tau_{2}(1)-T_{z} T_{\rho_{2}} \tau_{2}(1) & =p\left\{\rho_{1} T_{z} T_{\rho_{1}} \eta(1)+(1-\theta)\left[T_{z} T_{\rho_{1}} Q_{2}(1)\right.\right. \\
& \left.\left.-T_{z} T_{\rho_{2}} Q_{2}(1)-\left(T_{z} T_{\rho_{1}} Q_{1}(1)-T_{z} T_{\rho_{2}} Q_{1}(1)\right)\right]\right\} \\
& +q(1-\theta)\left\{m(0)\left[T_{z} T_{\rho_{1}} f * g(1)-T_{z} T_{\rho_{2}} f * g(1)\right]\right. \\
& \left.-m_{1}(0)\left[T_{z} T_{\rho_{1}} f(1)-T_{z} T_{\rho_{2}} f(1)\right]\right\} .
\end{aligned}
$$

Remember that $\rho_{1}=1$ and $\rho_{2}=q$. Substitute (28) and (33) into (22), the renewal equation then follows by inverting the p.g.f. of $\hat{m}(z)$ immediately.

Now we only need to show that the renewal Equation (23) is defective. In fact, by the positive relative safety condition we deduce that

$$
p \sum_{k=0}^{\infty} \zeta(k)=p \sum_{k=0}^{\infty} \sum_{j=k+1}^{\infty} f * g(j)=p\left(\mu_{F}+\mu_{G}\right)<1
$$

and theorem is proved.

We remark that explicit solution of the defective renewal equation (23) can be obtained by using results in $\mathrm{Li}$ [10] (Section 3) or $\mathrm{Wu}$ and $\mathrm{Li}$ [11] (Section 2 ). In the special case when $\theta=1$, the risk model studied in the present paper degenerates to the classical compound binomial risk process, and the only difference is that the claim amount variable is $X_{i}+Y_{i}$. Let $\theta=1$ in (23), the result coincides with Theorem 4.1 in [12] with some modifications. 


\section{Geometric Claim Amount Distribution}

In this section we assume that main claims and by-claims are both geometrically distributed with $f(x)=p_{1} q_{1}^{x-1}, g(x)=p_{2} q_{2}^{x-1}, x \in \mathbb{N}^{+}$for $0<q_{i}<1$ and $p_{i}=1-q_{i}, i=1,2$. It can be easily verified that

$$
\begin{gathered}
f * g(k)=\frac{p_{1} p_{2}\left(q_{2}^{k-1}-q_{1}^{k-1}\right)}{q_{2}-q_{1}}, \quad k=2,3, \cdots, \\
f * g * g(k)=\frac{p_{1} p_{2}^{2}\left\{\left[(k-2) q_{2}-(k-1) q_{1}\right] q_{2}^{k-2}+q_{1}^{k-1}\right\}}{\left(q_{2}-q_{1}\right)^{2}}, \quad k=3,4, \cdots .
\end{gathered}
$$

From (21) we know that

$$
\hat{m}(z)=\frac{\left(z-\rho_{1}\right)\left(z-\rho_{2}\right)\left[T_{z} T_{\rho_{2}} T_{\rho_{1}} \tau_{2}(1)-T_{z} T_{\rho_{2}} \tau_{2}(1)\right]}{(z-q)[z-q-p \hat{f}(z) \hat{g}(z)]} .
$$

Noting that $\hat{f}(z)=\frac{z p_{1}}{1-z q_{1}}, \hat{f}(z) \hat{g}(z)=\frac{z^{2} p_{1} p_{2}}{\left(1-z q_{1}\right)\left(1-z q_{2}\right)}$, after some careful calculations we obtain

$$
T_{z} T_{1} f(1)-T_{z} T_{q} f(1)=\frac{p q_{1}}{\left(1-z q_{1}\right)\left(1-q q_{1}\right)},
$$

and

$$
T_{z} T_{\rho_{1}} f * g(1)-T_{z} T_{\rho_{2}} f * g(1)=\frac{p\left\{1-q q_{1} q_{2}-q_{1} q_{2}\left[1+q\left(p_{1}-q_{2}\right)\right] z\right\}}{\left(1-z q_{1}\right)\left(1-z q_{2}\right)\left(1-q q_{1}\right)\left(1-q q_{2}\right)} .
$$

Denote by

$$
\varrho(z)=\left(1-z q_{1}\right)\left(1-z q_{2}\right)(z-q)^{2}-p p_{1} p_{2} z^{2}(z-q),
$$

which is a polynomial of degree 4 with leading coefficient $q_{1} q_{2}$. Obviously, 1 and $q$ are two roots of $\varrho(z)$. Therefore, if $R_{1}$ and $R_{2}$ are another two roots, we can rewrite $\varrho(z)$ as

$$
\varrho(z)=q_{1} q_{2}(z-1)(z-q)\left(R_{1}-z\right)\left(R_{2}-z\right) .
$$

In what follows, we assume that $R_{1}$ and $R_{2}$ are distinct, by partial fraction we have

$$
\frac{1}{\left(R_{1}-z\right)\left(R_{2}-z\right)}=\sum_{i=1}^{2} \frac{a_{i}}{R_{i}-z}, \quad a_{1}=\frac{1}{R_{2}-R_{1}}, a_{2}=\frac{1}{R_{1}-R_{2}},
$$




$$
\begin{gathered}
\frac{z}{\left(R_{1}-z\right)\left(R_{2}-z\right)}=\sum_{i=1}^{2} \frac{b_{i}}{R_{i}-z}, \quad b_{1}=\frac{R_{1}}{R_{2}-R_{1}}, \quad b_{2}=\frac{R_{2}}{R_{1}-R_{2}}, \\
\frac{1-z q_{2}}{\left(R_{1}-z\right)\left(R_{2}-z\right)}=\sum_{i=1}^{2} \frac{c_{i}}{R_{i}-z}, \quad c_{1}=\frac{1-q_{2} R_{1}}{R_{2}-R_{1}}, \quad c_{2}=\frac{1-q_{2} R_{2}}{R_{1}-R_{2}} \\
\frac{\left(1-z q_{1}\right)\left(1-z q_{2}\right)}{\left(R_{1}-z\right)\left(R_{2}-z\right)}=q_{1} q_{2}+\sum_{i=1}^{2} \frac{d_{i}}{R_{i}-z}, \\
d_{1}=\frac{1-R_{1}\left[q_{1}+q_{2}-q_{1} q_{2} R_{1}\right]}{R_{2}-R_{1}}, d_{2}=\frac{1-R_{2}\left[q_{1}+q_{2}-q_{1} q_{2} R_{2}\right]}{R_{1}-R_{2}} .
\end{gathered}
$$

Multiplying both the denominator and numerator of (34) by $\left(1-z q_{1}\right)\left(1-z q_{2}\right)$, (33), (35) and (36) imply that

$$
\begin{aligned}
\hat{m}(z)= & \frac{p q(1-\theta)}{q_{1} q_{2}\left(1-q q_{1}\right)\left(1-q q_{2}\right)}\left\{m ( 0 ) \left[\left(1-q q_{1} q_{2}\right) \sum_{i=1}^{2} \frac{a_{i}}{R_{i}-z}\right.\right. \\
& \left.\left.-q_{1} q_{2}\left(1+q\left(p_{1}-q_{2}\right)\right) \sum_{i=1}^{2} \frac{b_{i}}{R_{i}-z}\right]-m_{1}(0) q_{1}\left(1-q q_{2}\right) \sum_{i=1}^{2} \frac{c_{i}}{R_{i}-z}\right\} \\
& +\frac{p}{q_{1} q_{2}}\left(q_{1} q_{2}+\sum_{i=1}^{2} \frac{d_{i}}{R_{i}-z}\right) \hat{\delta}(z),
\end{aligned}
$$

where

$\hat{\delta}(z)=T_{z} T_{1} \eta(1)+(1-\theta)\left[T_{z} T_{1} Q_{2}(1)-T_{z} T_{q} Q_{2}(1)-\left(T_{z} T_{1} Q_{1}(1)-T_{z} T_{q} Q_{1}(1)\right)\right]$,

and the corresponding inverse image $\delta(u)$ is determined by $(24)$.

Inverting the p.g.f. in (37) gives an explicit expression for $m(u)$ as follows:

$$
\begin{aligned}
m(u)= & \frac{p q(1-\theta)}{q_{1} q_{2}\left(1-q q_{1}\right)\left(1-q q_{2}\right)}\left\{m ( 0 ) \left[\left(1-q q_{1} q_{2}\right) \sum_{i=1}^{2} a_{i} R_{i}^{-(u+1)}\right.\right. \\
& \left.-q_{1} q_{2}\left(1+q\left(p_{1}-q_{2}\right)\right) \sum_{i=1}^{2} b_{i} R_{i}^{-(u+1)}\right] \\
& \left.-m_{1}(0) q_{1}\left(1-q q_{2}\right) \sum_{i=1}^{2} c_{i} R_{i}^{-(u+1)}\right\} \\
& +\frac{p}{q_{1} q_{2}}\left[q_{1} q_{2} \delta(u)+\sum_{i=1}^{2} d_{i} \sum_{j=0}^{u} \delta(u-j) R_{i}^{-(j+1)}\right]
\end{aligned}
$$


Example. Let $\omega(x, y)=1$, then the Gerber-Shiu function $m(u)$ and $m_{1}(u)$ reduces to the ruin probabilities $\psi(u)$ and $\psi_{1}(u)$, respectively. Assume that $p=0.25, p_{1}=0.6$ and $p_{2}=0.75$. It is easy to check that the net profit condition holds. Now in this case, we obtain $R_{1}=6.1568433$ and $R_{2}=1.2181567$. The initial value $\psi(0)$ and $\psi_{1}(0)$, given in (12) and (13) respectively, are also obtained in Table 1.

Figure 1 gives the ruin probabilities $\psi(u)$ in Example 1, for fixed value $\theta=0,0.25,0.5,0.75,1$ and different initial values of $u=0,1,2 \ldots, 12$. It shows that these probabilities decrease generally as the initial surplus $u$ increases. Moreover, with fixed $u$, ruin probabilities increase as $\theta$ increases, which is consistent with our intuitive knowledge.

Table 1: $p=0.25, p_{1}=0.6, p_{2}=0.75$.

\begin{tabular}{cccccc}
\hline$\theta$ & 0 & 0.25 & 0.5 & 0.75 & 1 \\
\hline$\psi(0)$ & 0.5185185 & 0.5666667 & 0.6060606 & 0.6388889 & 0.6666667 \\
$\psi_{1}(0)$ & 0.6666667 & 0.7000000 & 0.7272727 & 0.7500000 & 0.7692308 \\
\hline
\end{tabular}

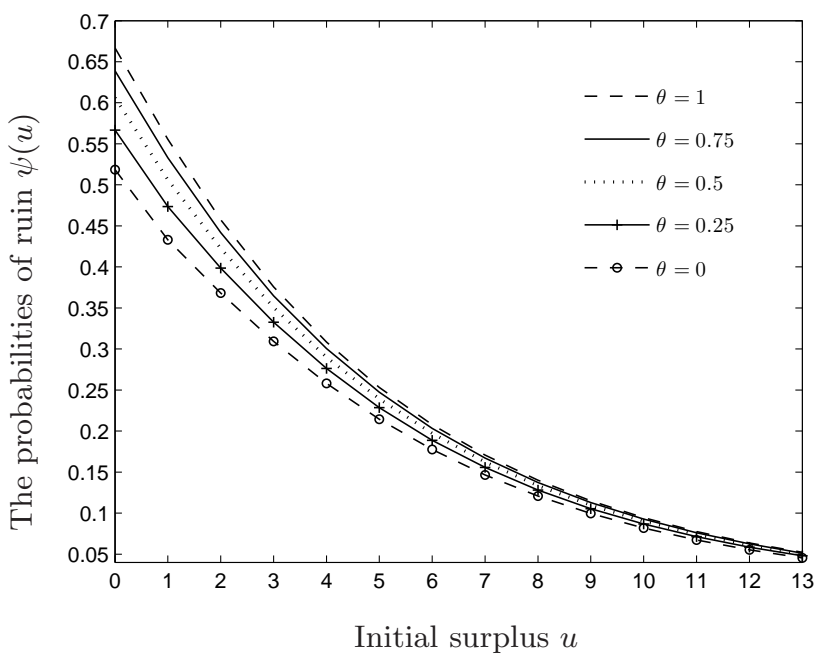

Figure 1: $p=0.25, p_{1}=0.6, p_{2}=0.75$. 


\section{Conclusions}

In this paper a framework of time-correlated claims is proposed by introducing two kinds of dependent claims, namely main claims and by-claims, and allowing possible delays of the occurrence of the by-claims. Some analytic techniques are applied to study the Gerber-Shiu function. We show that the generating function and defective renewal equation for the Gerber-Shiu function can be obtained. When the claim amounts follow geometric distribution, the explicit expression for the Gerber-Shiu function is also derived. The work of this paper can be regarded as an extension to the prior work on time-correlated claims studied by Xiao and Guo [5] and the references therein.

\section{Acknowledgements}

This research was supported by the Ministry of Education of Humanities and Social Science Project (15YJC910001) and Program for Liaoning Excellent Talents in University (LR2014031).

\section{References}

[1] H.U. Gerber, Mathematical fun with the compound binomial process, Astin Bull., 18 (1988), 161-168.

[2] G.E. Willmot, G.E. Ruin probabilities in the compound binomial model, Insurance Math. Econom., 12 (1993), 133-142.

[3] S. Cheng, H.U. Gerber, E.S.W. Shiu, Discounted probabilities and ruin theory in the compound binomial model, Insurance Math. Econom., 26 (2000), 239-250.

[4] K.C. Yuen, J. Guo, Ruin probabilities for time-correlated claims in the compound binomial model, Insurance Math. Econom., 29 (2001), 47-57.

[5] Y. Xiao, J. Guo, The compound binomial risk model with time-correlated claims, Insurance Math. Econom., 41 (2007), 124-133.

[6] X. Wu, S. Li, On a Discrete Time Risk Model with Delayed Claims and a Constant Dividend Barrier, Insur. Mark. Co.: Anal. Actuar. Comput., 3 (2012), 50-57.

[7] X. Wu, K.C. Yuen, A discete time risk model with interaction between classes of business, Insurance Math. Econom., 33 (2003), 117-133.

[8] H.U. Gerber, E.S.W. Shiu, On the time value of ruin, North Am. Actuar. J., 2 (1998), 48-78.

[9] S. Li, On a class of discrete time renewal risk models, Scand. Actuar. J., (2005), 241-260,

[10] S. Li, Distributions of the surplus before ruin, the deficit at ruin and the claim causing ruin in a class of discrete time risk models, Scand. Actuar. J., (2005), 271-284. 
[11] X. Wu, S. Li, On the discounted penalty function in a discrete time renewal risk model with general interclaim times, Scand. Actuar. J., (2009), 281-294.

[12] K.P. Pavlova, G.E. Willmot, The discrete stationary renewal risk model and the GerberShiu discounted penalty function, Insurance Math. Econom., 35 (2004), 267-277. 